\title{
STRUCTURAL CONDITION ASSESSMENT OF SEWERS AND ITS APPLICATION ON A CASE STUDY IN TOULOUSE, FRANCE
}

\author{
GEORGIA KANELLAKI $^{1}$, MARTA DEL PALACIO ${ }^{1} \&$ IDRISS BENSLIMANE $^{2}$ \\ ${ }^{1}$ Infraneo, Madrid, Spain \\ ${ }^{2}$ Infraneo, Paris, France
}

\begin{abstract}
Urban densification and transformation of cities have induced an interconnection and superposition between new and old structures. Thus, it is important to verify the structural compatibility and to evaluate the risk induced on the underground structures. The goal is to avoid generating an irreversible dysfunction with disproportionate costs on urban functioning vital elements, such as wastewater and rainwater collectors. These structures are frequently impacted by current construction projects, such as tunnels, underground parking and buildings. For this reason, their resistance should be assessed. Meanwhile, most of the sewage systems of metropolitan cities have reached their design working life or have been severely damaged. Structural health monitoring of collectors is therefore an ongoing field and a useful solution to extend service life of the structures. This paper aims to provide some insights toward structural condition assessment methods of sewage systems, such as visual inspection, radar auscultation, internal hydraulic jacking tests and modelling of the structural stability due to external parameters. Furthermore, a case study of a $150 / 100 \mathrm{~cm}$ ovoid-shaped combined sewer in Toulouse, France, will be analysed applying the aforementioned methods. It is a non-reinforced concrete structure which collects wastewater and rainwater from three main conduits of the city upstream of the purification station. In regard to the tunnelling works for the metro passage underneath the collector, it was necessary to assess the impact of this operation on its integrity. 3D soil-structure interaction model has been performed using the finite element method. Structure parameters and hardening soil ones have been given according to the diagnostic. The results of numerical simulation are given in this paper and they were used as constraints for the tunnelling operation.

Keywords: structural condition assessment, structural auscultation, underground structures, sewage systems, tunnel construction impact, internal hydraulic jacking tests.
\end{abstract}

\section{INTRODUCTION}

Modern urban planning imposes the superposition between old and new infrastructures. Thus, it is indispensable to evaluate the health condition of the existing structures and to verify their stability due to external parameters. Sewage structures are key-elements in the functioning of daily life in cities. They are underground structures the majority of which dates back from the beginning of the 20th century in European metropolitan cities. Most of the times, they have reached their service life or have been seriously damaged. Meanwhile, they are often impacted by the construction of new infrastructures like tunnels, underground parking, buildings, road backfills, etc. Any sever dysfunction of the structure could have harmful consequences, like flooding or pollution of the groundwater. Structural condition assessment of collectors is, therefore, a field that water and sewage stakeholders have developed the last decades in order to control the health state of their structures and to privilege trenchless maintenance works. Sewage stakeholders have classified the collectors as visitable, semi-visitable and non-visitable and they have adapted the types of auscultation depending on the dimension of the pipeline.

This paper aims to offer some insights of structural condition assessment of visitable collectors. Typical auscultation methods like visual inspection and ground penetration radar 
(GPR), but also innovative solutions, explicitly developed in the context of sewage auscultation, as internal hydraulic jacking tests will be presented. The goal is to estimate qualitatively and quantitatively the current state of the structure and the ground casing and bedding the collector. These two entities often work as a system and influence the stability of the sewer. Auscultations results are used to carry out a diagnostic study of the structure and according to the conclusions propose recommendations of maintenance works. They can also be the input data to assess the stability of the structure due to construction of new projects in the proximity.

In this context, a case study of a 150/100 cm ovoid-shaped combined sewer collector in Toulouse, France, will be analysed applying the aforementioned methods. It is a representative inspection, as the collector has been subjected to multiple types of emergency diagnostics due to collapsing of a part of the duct next to the national highway. In addition, metro construction underneath the collector is expected several meters upstream the collapsing zone, so the stability of the structure should be evaluated. This example will allow understanding how conclusions are extracted from each auscultation and are combined to define a degradation state of the structure. Subsequently, these conclusions for the structure and the soil will be interpreted into material properties to produce a finite element model and to define the thresholds of the settlements curve imposed by the tunnel on the structure.

\section{STRUCTURAL CONDITION ASSESSMENT IN SEWAGE SYSTEMS}

Diagnosis studies use different methods, such as visual inspection, non-destructive auscultations, core sampling and geotechnical essays, to divide the surveyed length of the collector into homogeneous sections of similar characteristics. Subsequently, the sewer and its surrounding ground are classified in an acceptable, medium and poor state according to diagnosis results. In this study, only non-destructive methods will be assessed.

\subsection{Visual inspection}

Visual inspection is carried out within the context of technical standards and recommendations, based on experience feedback [1]. This experience has grouped the degradations of sewers into five distinct families, ranked in descending order of potential structural risk, and therefore in relation to restructuring needs: breaks, deformations, leaks, punctual anomalies, degradation of coatings. According to the frequency and the significance of anomalies, the condition of the structure can be classified good, medium or poor. The visual inspection frequently allows to do pre-diagnostics studies in order to determine the cause of the damage and the adequate type of auscultation for the system soil/structure. Meanwhile this method is not sufficient enough to do a complete diagnosis.

\subsection{Ground penetration radar auscultation}

Radar auscultations allow detecting the existence, the position, the depth or the nature of an object through the study of the reflection of electromagnetic waves. GPR is a non-destructive technique (NDT) which detects interfaces between materials (structure, geological levels) or heterogeneity presence (cavities, flow paths, etc.). The wave frequencies used for this kind of auscultations are between $10 \mathrm{MHz}$ to $5 \mathrm{GHz}$ [2]. Radar auscultation is feasible by surface or from inside buried structures. Underground infrastructures are studied in a frequency range of $100 \mathrm{MHz}$ to $1 \mathrm{GHz}$ which is used to investigate on the meter scale, but specific information acquisition as concrete reinforcement detection (investigation depth less than $0.5 \mathrm{~m}$ ) uses 
frequencies until $2 \mathrm{GHz}$. Sewage systems condition assessment uses widely GPR auscultations to determine information about:

- Structure: Nature of structure (concrete reinforced or non-reinforced, masonry), thickness, quality and reinforcing bars location.

- Interface between structure and soil: Presence of timbering and metal frames from construction shielding and quality assessment (voids presence, decompressions, detachments, etc.).

- Ground (cover/casing/bedding soil): Ground nature, geological levels and quality of the ground around the sewer, determination of nearby infrastructures presence (urban networks, underground structures, foundations, etc.).

\subsection{Internal hydraulic jacking tests}

Internal hydraulic jacking tests aim to calculate the soil micro-deformation modulus and determine a quality index for the underground structure by measuring a global stiffness of the system soil-structure. The jacking device contains a hydraulic jack and three rods placed between the walls (with a longitudinal spacing of approximately an equivalent diameter of the section) and just below the vault. The principles of the test are presented in Fig. 1(a). This auscultation method has been developed in France. Water and wastewater stakeholders have widely acknowledged this method during the last two decades.

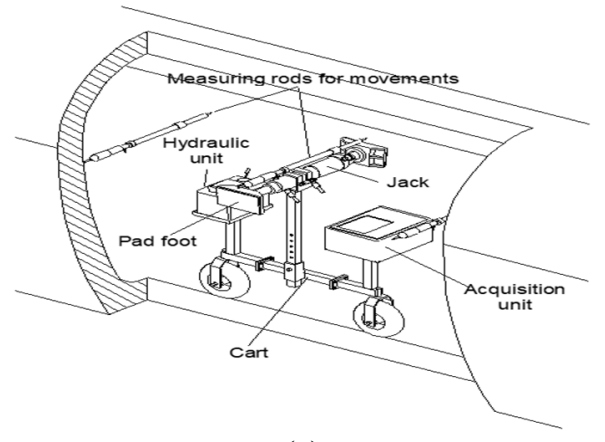

(a)

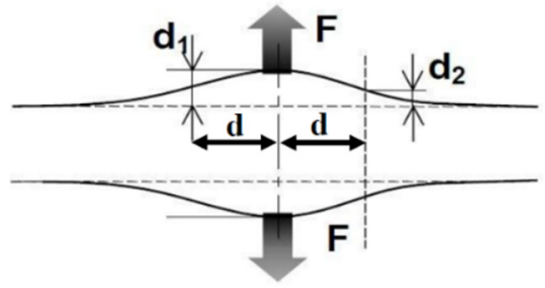

(b)

Figure 1: (a) Jacking device presentation; and (b) internal hydraulic jacking tests principles.

The test consists in applying an ovalization loading on the conduit by means of a horizontal jack. Three displacements are extracted with help of the measuring rods, one on level of the jack and two others at a certain distance from this last one (Fig. 1(b)). The measures remain always in the elasticity domain (micro-deformations). Each test has two loading cycles and each parameter is measured and calculated twice. The values obtained are generally averaged to constitute an aggregated result.

The calculation is inspired by a simple continuous beam on elastic supports (spring stiffness $k$ ), which represents the system soil-structure [3]. The stiffness of this system in longitudinal direction $\left(K_{g}\right)$ is a combination of the bending rigidity of the structure $(E I)$ and the modulus of reaction of the ground $(k)$. Increasing relative stiffness of the soil results in a concentration of the deformation around the loading application point. Thus, the test consists in assessing simultaneously the total stiffness of the system (eqn (1)) and the localisation of 
the response (eqn (2)) to determine the stiffness of the beam and its supports distinctly, by using the displacements measurements $\left(d_{1}, d_{2}\right)$ and the applied load $(F)$.

Global stiffness:

$$
K_{g}=\frac{F}{d_{1} / 2}
$$

Deformation transmission ratio:

$$
\Omega=\frac{d_{2}}{d_{1}}
$$

The goal of this procedure is to achieve decoupling the system, i.e. to represent the factors $E I$ and $k$ with functions related directly to the measured values of $K_{g}$ and $\Omega$. Decoupling of the system is done with the help of a finite element (FE) model and iterative calculations in order to obtain a polynomial expression for each variable. The model is based on the assumptions that the two parameters are distinguished and their mechanical behaviour is elastic, linear, homogeneous and isotropic. Each material is characterised by a longitudinal deformation modulus $\left(E_{i}\right)$ and a Poisson coefficient $(v)$. The interface between the two materials does not consider slippage and detachment and the only charge considered is the internal jack load. The iterative procedure calculates the theoretical stiffness and transmission rate for eleven representative cases, from the unsupported structure $\left(K_{0}\right.$, without soil contribution) until a system with a very compact soil contribution. The resolution is based on a dimensionless problem that defines the curves of the soil and structure modulus in relation to total stiffness in function of the transmission ratio $\left(E_{c} / K_{g}-\Omega\right.$ and $\left.E_{s} / K_{g}-\Omega\right)$. Afterwards, the cubic degree polynomial adjustment of these curves allows obtaining their analytical resolution (eqns (3) and (4)).

$$
\begin{aligned}
& \frac{E_{s}}{K_{g}}=f_{1}\left(\frac{\Omega}{\Omega_{0}}\right) \cong \sum_{n_{1}}^{k=0} \alpha_{k}\left(\frac{\Omega}{\Omega_{0}}\right)^{k} \\
& \frac{K_{C}}{K_{g}}=f_{2}\left(\frac{\Omega}{\Omega_{0}}\right) \cong \sum_{n_{2}}^{k=0} \bar{\alpha}_{k}\left(\frac{\Omega}{\Omega_{0}}\right)^{k}
\end{aligned}
$$

where $E_{s}$ and $K_{c}$ are the soil and structure stiffness respectively, $k$ is the polynomial expression degree (limited at 3) and $\alpha_{0}, \alpha_{1}, \alpha_{2}, \alpha_{3}, \bar{\alpha}_{0}, \bar{\alpha}_{1}, \bar{\alpha}_{2}, \bar{\alpha}_{3}$ are the polynomial constants. $\Omega_{0}$ is the transmission ratio for the case of the unsupported structure. The quality index of the structure for each measure is obtained as $I Q=K_{c} / K_{0}$ where $K_{0}$ is the unsupported structure stiffness.

The transmission ratio takes its maximal value for the case of the unsupported structure $\left(\Omega_{0}\right)$, beyond this value, decoupling is not possible. This value $\Omega_{\max }$ depends exclusively on structure's thickness and geometry. The relation $E_{c} / K_{g}$ is an increasing function, while $E_{s} / K_{g}$ is a decreasing function which depends quasi-linearly on $\Omega$ in a wide range of variation, except for small values of the three-dimensional effect (Fig. 2). This case reflects rather the compression (crushing) of the collector in the thickness direction than an overall response of the structure in bending. It should therefore also be possible to deduce a minimum value $\Omega_{\text {min }}$ below which the test no longer falls within the proposed interpretation scheme.

The curves tendency allow a qualitative interpretation of the tests results for a measurements section. When $K_{g}$ and $\Omega$ vary in the same direction, sewer's stiffness follows total stiffness evolution. For the opposite case, it is observed the same behaviour for soil stiffness. Thus, it is possible to distinguish zones that qualitatively have different geometrical or mechanical characteristics. 


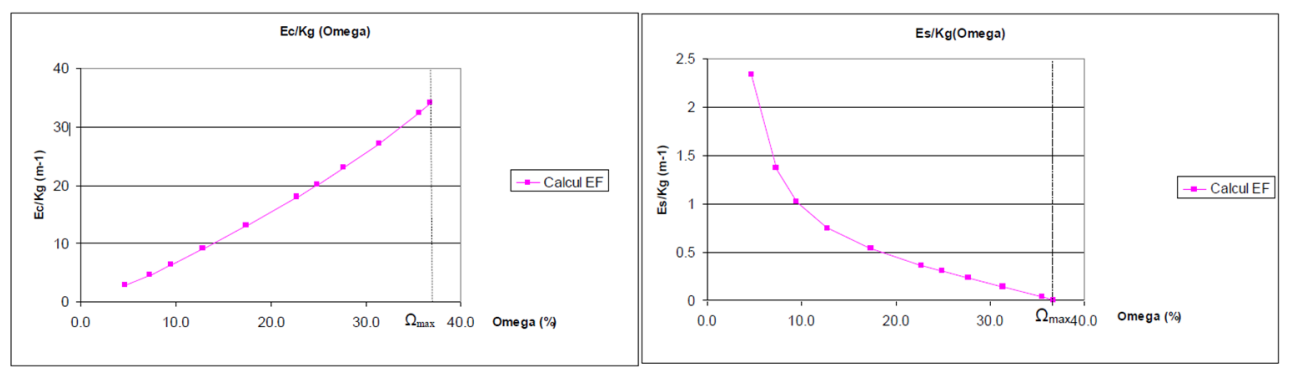

Figure 2: Evolution of $E_{c} / K_{g}$ and $E_{s} / K_{g}$ in relation to $\Omega$.

The calculated soil modulus $\left(E_{S}\right)$ corresponds to the microdeformation domain of deformations (less than $10^{-5}$ ). The relationship between the soil modulus and the deformation domain is defined as logarithmic (Fig. 3). Using the results of the internal hydraulic jacking tests, the modulus $\left(E_{s}\right)$ is translated into the deformation domain of underground structures (tunnels) as its value divided by 2 or 3 .

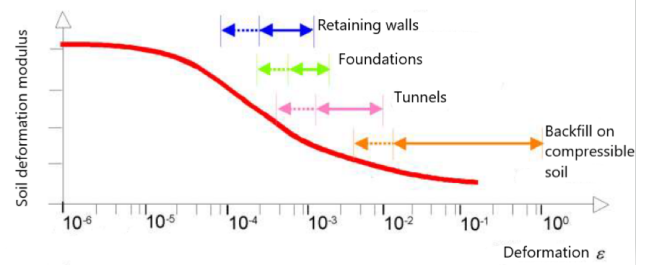

Figure 3: Evolution of the soil modulus in relation to the referenced deformation domain [4].

\section{ABC COLLECTOR IN TOULOUSE}

$\mathrm{ABC}$ collector in Toulouse, France is a representative study case of sewage systems condition assessment. It is a $150 / 100 \mathrm{~cm}$ ovoid-shaped combined sewer built with non-reinforced concrete. It collects wastewater and rainwater from three main conduits of the city upstream the purification station, it is thus under important hydraulic charge and should be rarely nonoperational. The collector has been subjected to multiple types of emergency diagnostics on different parts along its length, due to collapsing of a part of the conduit. In addition, the passage of the upcoming metro line 3 and the fragility of the structure pointed out the necessity of a tunnelling impact study to determine the admissible settlement curve induced by the metro construction underneath the sewer (Fig. 4).

\subsection{Collapsing of the collector, structural condition assessment and rehabilitation}

In February 2021, the sewer subjected to an emergency diagnostics on a $450 \mathrm{~lm}$ part of the structure linear (RV-1 to RV3 and RV3.4 to RV4 $+40 \mathrm{~m})$ due to collapsing of a part of the conduit (RV3.1 to RV3.4), situated next to the national highway. The structural condition assessment included visual inspection, internal hydraulic jacking tests, radar auscultation, core sampling and resistance tests of the structure and the soil behind. Visual inspection revealed poor degradation of the structure. Thus, radar auscultation and internal hydraulic jacking tests allowed investigating further the cause of the collapsing. 


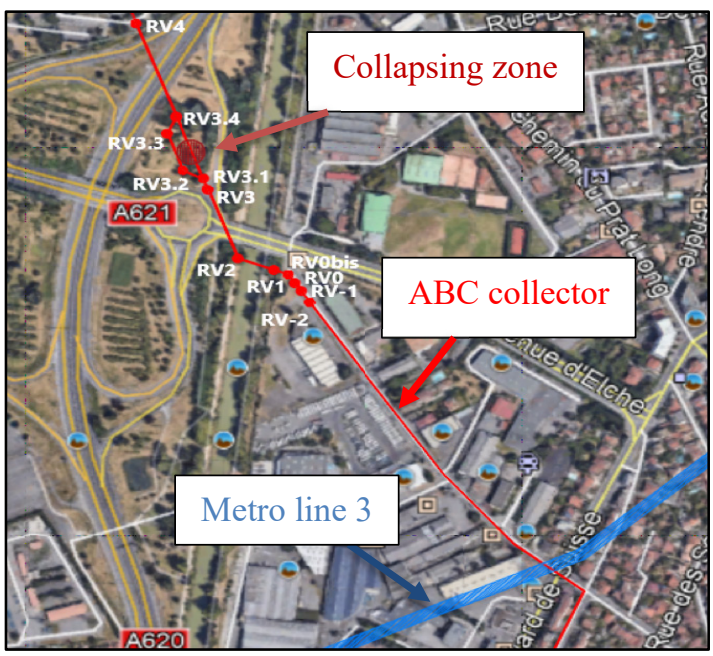

Figure 4: Disposition of the ABC collector in the studied zone.

Radar devices took measures from the intrados of the sewer on the vault and the base of the sidewalls (Fig. 5(a)). Radar auscultation determined the quality of the soil, the structure and the interface between them. Radar survey indicated heterogeneities at the interface and in the ground on both sides and the vault of the collector for the zone next to the collapsing (Fig. 5(b)). These results can be correlated with the presence of water leakage due to structure degradation. These water exfiltration are the cause of soil decompression by entrainment of fines. In addition, the survey estimated the medium thickness of the structure to $25 \mathrm{~cm}$.

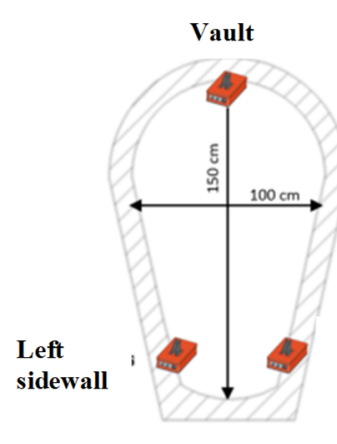

(a)

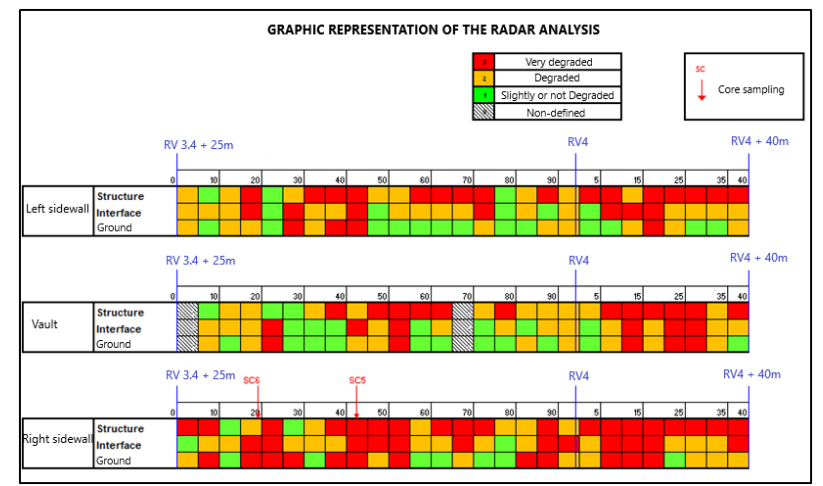

(b)

Figure 5: (a) Radar position; and (b) Results of the radar survey for the part between $\mathrm{RV} 3.4+25 \mathrm{~m}$ and RV4 $+40 \mathrm{~m}$ of ABC collector.

Internal hydraulic jacking tests obtained initially the total stiffness and the transmission ratio of the system (Fig. 6). A model of the ovoid structure was produced according to its 


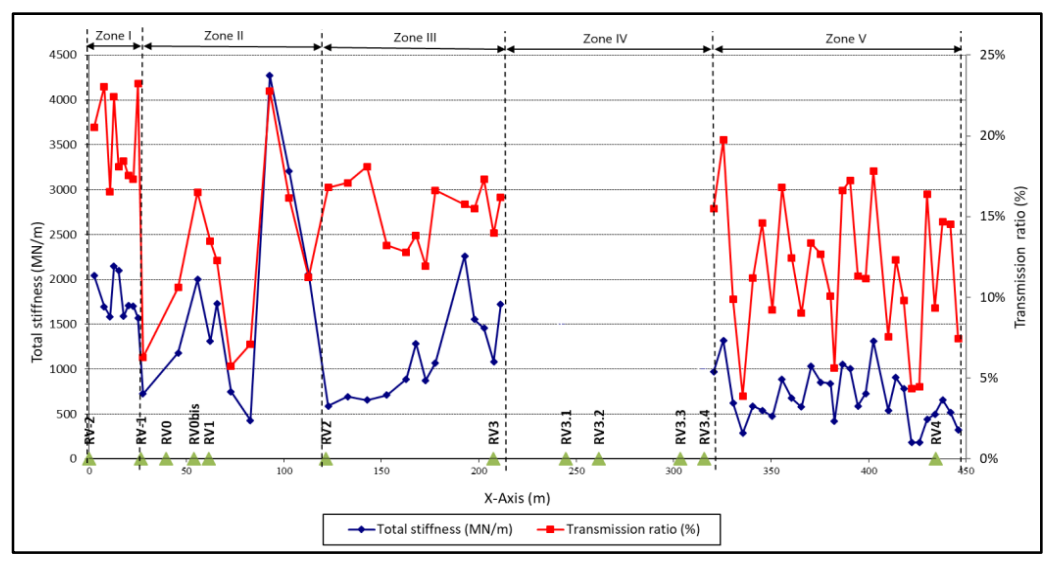

Figure 6: Internal hydraulic jacking tests measures.

Table 1: Zone definition for internal hydraulic jacking tests measurements and mean results.

\begin{tabular}{|c|c|c|c|c|c|}
\hline Zone & $\begin{array}{c}\text { Total stiffness } \\
\left(K_{g}\right)\end{array}$ & $\begin{array}{c}\text { Transmission } \\
\text { ratio }(\Omega)\end{array}$ & $\begin{array}{c}\text { Covering } \\
\text { high }\end{array}$ & $\begin{array}{c}\text { Soil stiffness } \\
\left(E_{s}\right)\end{array}$ & $\begin{array}{c}\text { Quality index } \\
(\mathrm{IQ})\end{array}$ \\
\hline I & $155-200 \mathrm{MN} / \mathrm{m}$ & $18-24 \%$ & $3.5 \mathrm{~m}$ & $210 \mathrm{MN} / \mathrm{m}$ & $60 \%$ \\
\hline II & $500-4200 \mathrm{MN} / \mathrm{m}$ & $5-24 \%$ & $3-9 \mathrm{~m}$ & $670 \mathrm{MN} / \mathrm{m}$ & $33 \%$ \\
\hline III & $600-2300 \mathrm{MN} / \mathrm{m}$ & $12-18 \%$ & $9 \mathrm{~m}$ & $340 \mathrm{MN} / \mathrm{m}$ & $33 \%$ \\
\hline IV & Not auscultated & Not auscultated & - & - & \\
\hline V & $100-1200 \mathrm{MN} / \mathrm{m}$ & $3-21 \%$ & $3.6 \mathrm{~m}$ & $318 \mathrm{MN} / \mathrm{m}$ & $16 \%$ \\
\hline
\end{tabular}

geometrical characteristics and a theoretical modulus of $35 \mathrm{GPa}$. The parameters of the unsupported structure were determined to $\Omega_{0}=24 \%$ and $K_{0}=2194 \mathrm{MN} / \mathrm{m}$. The analysis pointed out five zones where the characteristics could be grouped qualitatively (Table 1).

The decoupling of the system complemented radar results by defining a poor to medium state of the structure in the zone around the collapsing (Zone III and V, Fig. 7). In the zone I, more than $100 \mathrm{~m}$ upstream the collapse, the structure and ground charts present a more homogenous behaviour. The structure is in a medium to acceptable state, while the soil is loose to compact (Fig. 8). The difference of the covering high of the structure due to the highway backfills disposition (escalation from $3 \mathrm{~m}$ to $9 \mathrm{~m}$ at RV1 $+40 \mathrm{~m}$ ) produce heterogeneous results concerning the compactness of the soil in the zone II.

\subsection{Metro construction impact on the structure: Tunnelling impact study}

As the structure is sensible, it was indispensable to assess the impact induced by the tunnel construction and to define thresholds for its integrity. The metro line 3 will be constructed underneath the collector. The sewer will be impacted by the settlements of the bedding ground due to its deconfinement during the passage of the tunnel boring machines (TBM). That procedure induces tensile stresses at the invert. The goal was to determine the acceptable thresholds for the sewer in terms of maximum settlement / maximum slope, induced by the TBM underneath the structure. These thresholds are set so as not to exceed the serviceability limit of the material constituting the collector structure. In the case of exceeding these 


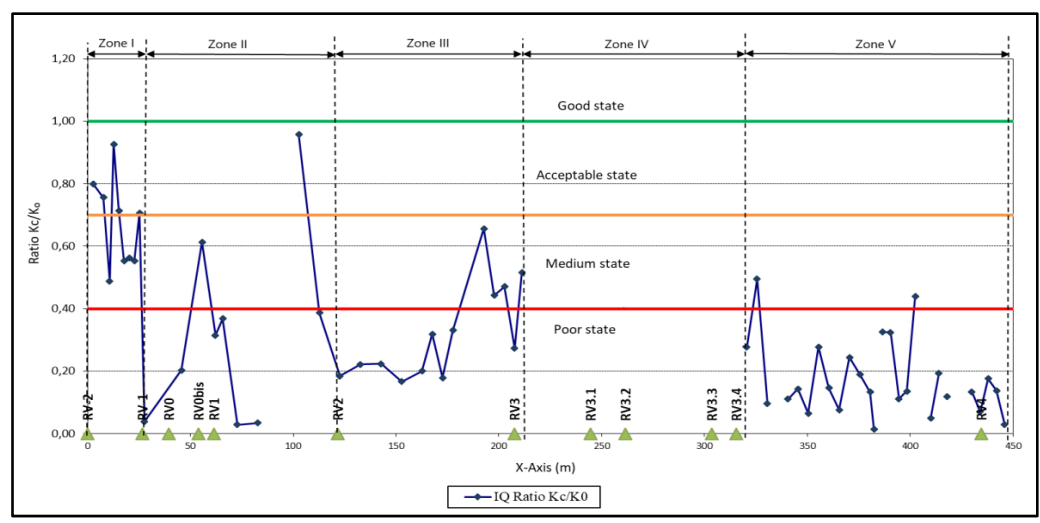

Figure 7: Quality index resulted from internal hydraulic jacking tests results.

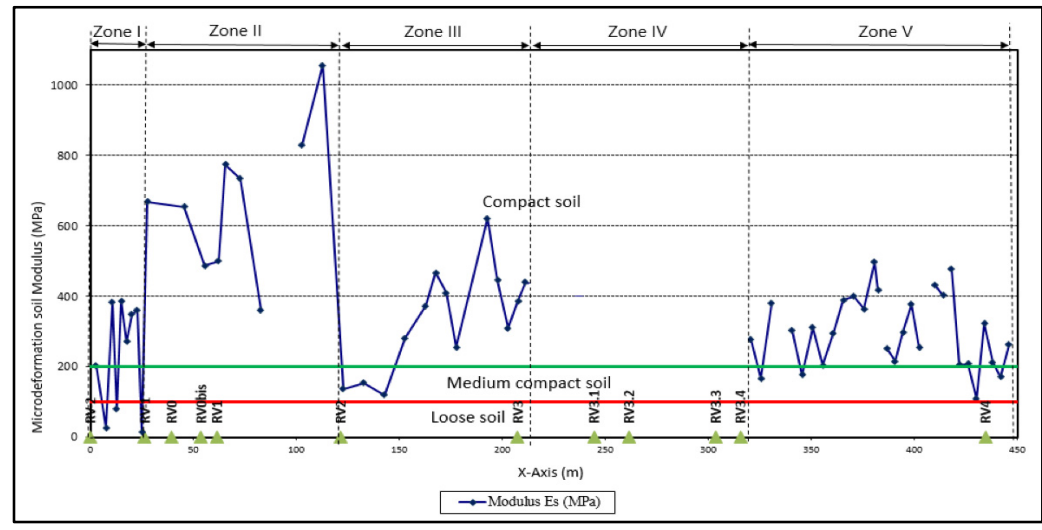

Figure 8: Soil modulus resulted from internal hydraulic jacking tests results.

admissible settlements, a reinforcement solution should be applied on the structure. A 3D soil-structure interaction model was performed using CESAR 3D LCPC software [5] in order to produce the tunnelling impact study. The structural condition assessment on the collector a few linear meters upstream the tunnelling impact zone was an important tool for the definition of the material properties of the structure and the ground.

\subsubsection{Settlement curve underneath the collector}

The settlements induced underneath the structure were defined based on recent bibliography. Most of the current research has focused on measuring and determining the settlements generated on the surface [6], which present the shape of a Gaussian curve. It has been observed that the settlements at a given depth follow this curve. The goal of an impact study is to evaluate the stresses generated at the underground structure from the existing empirical formulas for the determination of settlements and from numerical modelling methods. Peck's formula [7] is one of the most acknowledged to calculate le settlement curve of a tunnel (Fig. 9). According to this formula the displacement on each point of the transversal axis of the 


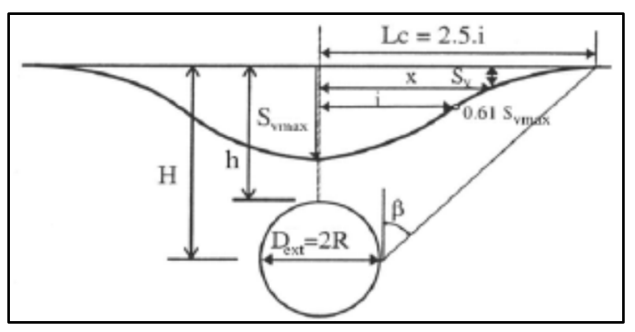

Figure 9: Settlement curve due to tunnel passage according to Peck's formula [7].

passage is a Gaussian curve (eqn (5)). It depends on the maximum settlement $\left(S_{v \max }\right.$, eqn (6)), the volume loss due to the excavation $\left(V_{L}\right)$, the tunnel diameter $\left(D_{e}\right)$ and depth $(\mathrm{H})$. The distance of the inflexion point from the centre of the hollow is determined in this case by the O'Reilly and New approach [8] and depends on a parameter (K) of the soil nature on each ground layer (eqn (7)).

$$
\begin{gathered}
S(x)=S_{\text {vmax }} e^{\left(-\frac{x^{2}}{2 i^{2}}\right)}, \\
S_{\text {vmax }}=0,313 \frac{V_{L} D_{e}^{2}}{(0,5 H)}, \\
i=K_{i} H_{i},
\end{gathered}
$$

where $K_{i}$ and $H_{i}$ the $\mathrm{K}$ parameter and the depth for each ground layer.

The effect of the impact depends on the relative position between the tunnel and the sewer. A transverse passage will produce displacements along the sewer and thus longitudinal stresses. A longitudinal passage will produce stresses in the cross section.

\subsubsection{Finite element model}

TBM will cross the sewer in an oblique angle (Fig. 4). The model considered that it is perpendicular to the collector in order to assess the case corresponding to the steepest Peck's curve slope. Several theoretical settlement curves of different amplitudes applied to the model, according to different excavation depths with maximum settlements of $5 \mathrm{~mm}, 10 \mathrm{~mm}$ and $15 \mathrm{~mm}$ (Fig. 10). TBM characteristics and geotechnical parameters were based on experience feedback report (REX) for the construction of the metro line B in Toulouse [9]. The excavation parameters $D_{e}=7.8 \mathrm{~m}$ and $H=7.54$ to $22.3 \mathrm{~m}$ and the settlements measured in the REX $\left(S_{v \max } \sim-3 \mathrm{~mm}\right)$ for a tunnel excavated with slurry pressure balanced (SPB) tunnel boring machines conditioned the variables $K=1.12$ and the loss of volume $V_{L}=0.20 \%$.

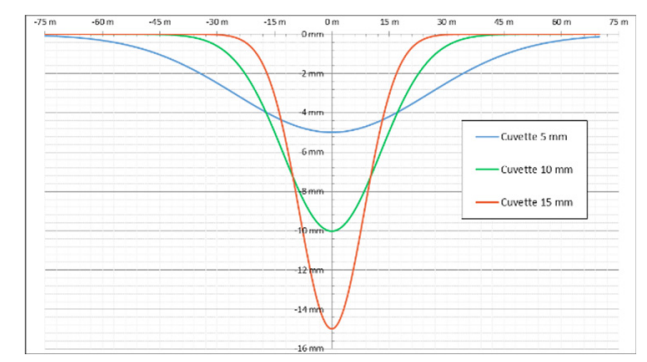

Figure 10: Figure settlement curves for maximum settlement of 5, 10, and $15 \mathrm{~mm}$. 


$$
\begin{gathered}
S_{v \max }=5 \mathrm{~mm}, H=22.3 \mathrm{~m}, i=0.12 \% 0, \\
S_{v \max }=10 \mathrm{~mm}, H=11.3 \mathrm{~m}, i=0.47 \% 0, \\
S_{v \max }=15 \mathrm{~mm}, H=7.54 \mathrm{~m}, i=1.07 \% 0 .
\end{gathered}
$$

The finite element model used the static soil-structure interaction theory. The calculations carried out without amplification coefficients of the loads so that the soil elasto-plastic behaviour is unaffected. The ultimate limit state (ULS) of the structure is obtained by applying the magnification factors to the results. 3D model represented a section of the soil around the sewer with a width of five equivalent diameters of the pipe (to neglect the edge effects) and a depth of $1 \mathrm{~m}$ underneath the invert base. It assessed only one quarter of the problem due to the symmetry of the settlement curve as well as the axial symmetry of the structure. The model only covered the longitudinal half of the settlement basin and the transverse half of the collector (Fig. 11). The length of the numerical models was set to $70 \mathrm{~m}$ (larger than half the width of the longest settlement basin) in order to neglect as much as possible the edge effects on the boundary conditions of the model. The boundary conditions defined as blocked displacements assigned to the sides of the soil volume.

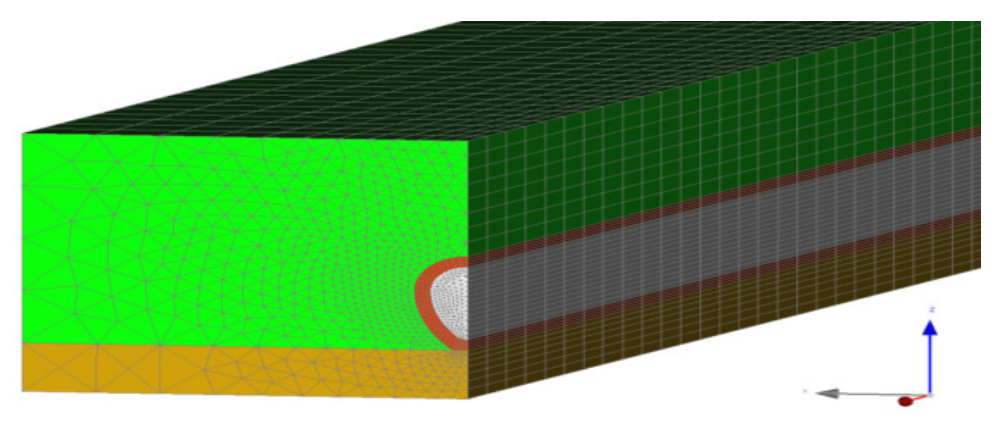

Figure 11: 3D mesh model.

3D model considered the structure as geometrically continuous. No defect, discontinuity or damage was taken into account. Thus, the mechanical properties of the materials describe a global behaviour and they were defined by the auscultations. The behavioural models considered for the materials are isotropic linear elasticity for the sewer and hardening soil model (HSM) [10] in elasto-plasticity for the soil (casing, cover, bedding and interface). Soil characteristics estimated from typical geotechnical parameters for each layer and the radar observations that characterised a terrain overly medium quality (Table 2). Soil loading modulus for the casing terrain determined by the internal hydraulic jacking tests results. It considered that the model was into the tunnelling domain of deformations. Compression essays produced on the core sampling of the structure and measured fluctuating resistances. Concrete thresholds were fixed by considering a conservative modulus of elasticity at $25 \mathrm{GPa}$ and calculating the associated compression and tensile resistances for SLS according to the EC2. The deformation threshold was determined on the basis of the AFTES recommendations [11], which indicate a limit of $0.3 \%$ at SLS for underground concrete networks. Taking into account the sensitive nature of sewerage networks (exfiltration), this value was limited to $0.15 \%$. 
Table 2: Geotechnical parameters of the model.

\begin{tabular}{|c|c|c|c|c|c|c|}
\hline \multirow{2}{*}{ Terrain } & \multicolumn{6}{|c|}{ Soil parameters } \\
\cline { 2 - 7 } & Nature & $\begin{array}{c}\text { Density } \\
(\gamma)\end{array}$ & $\begin{array}{c}\text { Poisson } \\
\text { coefficient }(v)\end{array}$ & $\begin{array}{c}\text { Cohesion } \\
\left(\mathrm{c}^{\prime}\right)\end{array}$ & $\begin{array}{c}\text { Friction } \\
\text { angle } \\
\left(\varphi^{\prime}\right)\end{array}$ & $\begin{array}{c}\text { Loading } \\
\text { Modulus } \\
\left(E_{50 \text { ref }}\right)\end{array}$ \\
\hline Casing & Backfills & $20 \mathrm{kN} / \mathrm{m}^{3}$ & 0.33 & $0 \mathrm{kPa}$ & $25^{\circ}$ & $50 \mathrm{MPa}$ \\
\hline Bedding & Alluvium & $20 \mathrm{kN} / \mathrm{m}^{3}$ & 0.33 & $10 \mathrm{kPa}$ & $30^{\circ}$ & $100 \mathrm{MPa}$ \\
\hline
\end{tabular}

The loads were applied in distinct phases according to the construction mode of the structure. The first phase consisted on the initialisation of the soil stresses by applying its self-weight. Afterwards, the phase of the collector construction followed, with the application of its own weight as well as the soil deconfinement forces around the structure $(\lambda=1$, collector built in trench). Finally, the excavation of the TBM under the collector was modelled. In this phase, the settlement (Gaussian curve) was imposed as a displacement on the z-axis on the ground one meter below the invert of the collector. This modelling convention took into account the possible influence of the presence of the structure for the reduction of the settlements generated by the excavation. In this phase, a hydrostatic pressure was also considered inside the collector to take into account the weight of the water in case the pipe is full at the time of the passage of the TBM (Fig. 12).

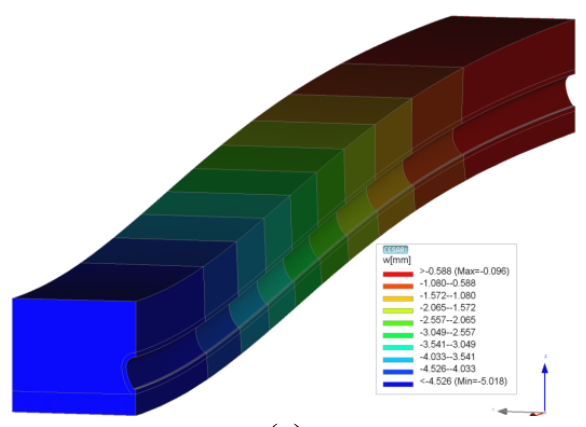

(a)

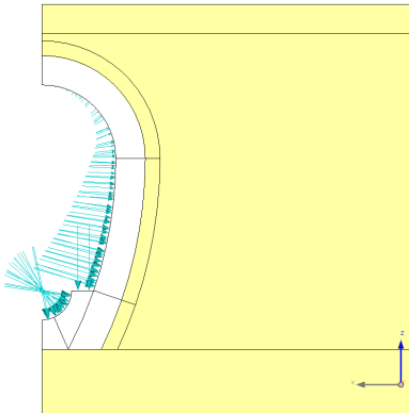

(b)

Figure 12: (a) Settlement curve imposed on the model; and (b) hydraulic load into the sewer.

Several models were carried out to apply the different theoretical settlements proposed. The maximum normal stresses generated in the collector in the longitudinal direction occur at the point of maximum settlement. The tensile stress is the dimensioning parameter as its threshold is exceeded when the maximum settlement of $10 \mathrm{~mm}$ is applied (Table 3). The

Table 3: Modelling results.

\begin{tabular}{|c|c|c|c|}
\hline Curve & $\mathrm{S}_{\mathrm{vmax}}=5 \mathrm{~mm}$ & $\mathrm{~S}_{\mathrm{vmax}}=10 \mathrm{~mm}$ & Thresholds \\
\hline Compression max $\sigma_{\mathrm{yy}}$ & $-0.283 \mathrm{MPa}$ & $-0.665 \mathrm{MPa}$ & $\sigma_{\mathrm{c} \mathrm{ELS}}=0.6 \mathrm{f}_{\mathrm{ck}}=-4.39 \mathrm{MPa}$ \\
\hline Tensile max $\sigma_{\mathrm{yy}}$ & $0.067 \mathrm{MPa}$ & $0.805 \mathrm{MPa}$ & $\sigma_{\mathrm{t} \text { ELS }}=0.6 \mathrm{f}_{\mathrm{ctm}}=0.68 \mathrm{MPa}$ \\
\hline Deformation max $\varepsilon_{\mathrm{yy}}$ & $0.01 \% \mathrm{o}$ & $0.07 \% \mathrm{o}$ & $0.15 \% \mathrm{o}$ \\
\hline
\end{tabular}




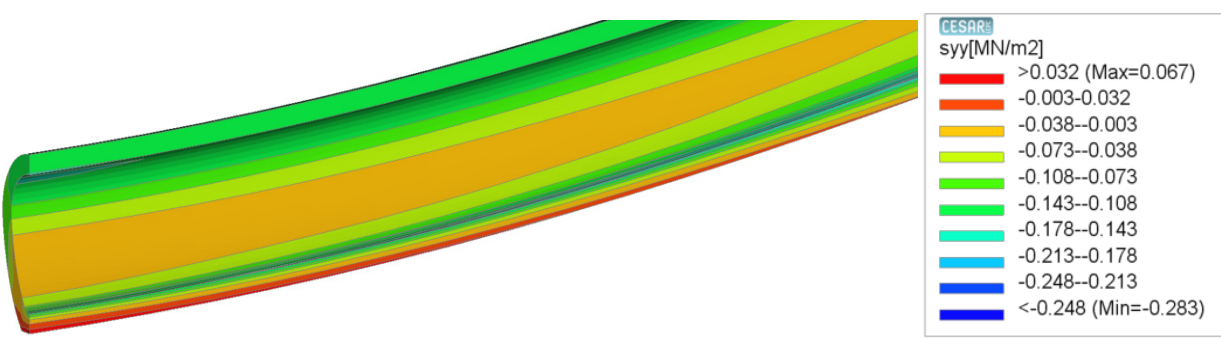

Figure 13: Normal stresses in the longitudinal direction (MPa) for the case of a $5 \mathrm{~mm}$ settlement curve.

threshold curve applied under the sewer is a maximum settlement of $5 \mathrm{~mm}$. For this case, the tensile stresses are located in the invert and compressive stresses in the vault. They extend over a length of about $15 \mathrm{ml}$ on either side of the tunnel axis (Fig. 13).

\section{CONCLUSION}

The paper provides a case study for the structural condition assessment of visitable sewers. It is based on existing methods like visual inspection and non-destructive auscultations, such as radar and internal hydraulic jacking tests, explicitly developed for this type of underground infrastructures. Through these methods a tunnelling impact study has been undertaken and the risk induced on the underground structures due to the construction of new metro lines has been determined. The admissible settlement curve imposed on the collector calculated by a soil-structure interaction finite element model. Calculated admissible settlements will serve as constraints for the tunnelling operation. Carrying out internal hydraulic jacking tests and visual inspection after the tunnel construction could allow to detect the evolution of the structure cracking and of the soil compactness. If necessary, a reinforcement solution should be applied.

\section{REFERENCES}

[1] Projet national rehabilitation des réseaux d'assainissement urbains (RERAU), Restructuration des collecteurs visitables, 2004.

[2] Quinta-Ferreira, M., Ground penetration radar in geotechnics: Advantages and limitations. World Multidisciplinary Earth Sciences Symposium (WMESS), 2018.

[3] Thepot, O., Prise en compte des caractéristiques en petites deformations des sols dans l'étude du comportement des collecteurs enterrés. Ecole Nationale des Ponts et Chaussées, 2004.

[4] Khiatine, M., Reiffsteck, P. \& Ramdane, B., Evaluation of dynamic soil properties for alluvial plain of Bejaia using field data and laboratory tests. Geotechnical and Geological Engineering, 37, pp. 4707-4730, 2019.

[5] CESAR 3D - LCPC, Computer software, itech, 2014.

[6] Viggiani, G. \& Soccodato, F.M., Predicting tunnelling-induced displacements and associated damage to structures. Rivista italiana di geotecnica, 4, 2004.

[7] Peck, R.B., Deep excavations and tunnelling in soft ground. 7th International Conference of Soil Mechanics and Foundations, pp. 226-290, 1969.

[8] O'Reilly, M.P. \& New, B.M., Settlements above tunnels in the United Kingdom: Their magnitude and prediction. Proc. Tunnelling, 82, pp. 387-400, 1982. 
[9] Vanoudheusden, E., Impact de la construction de tunnels urbains sur les mouvements de sol et bâti existant: Indice du mode de pressurisation du front. L'Institut National des Sciences Appliquées de Lyon, 2006.

[10] Schaz, T., Vermeer, P.A. \& Bonnier, P.G., The hardening soil model: Formulation and verification. Beyond 2000 in Computational Geotechnics - 10 Years of Plaxis, 1999.

[11] Recommendations de l'Association Française des Tunnels et de l'Espace Souterrain (AFTES), Tassements liés au creusement des ouvrages en souterrain, p. 13, 2018. 